\title{
Multidrug-resistant Acinetobacter baumannii infections in COVID-19 patients hospitalized in intensive care unit
}

\author{
Alessandro Russo ${ }^{1,2} \cdot$ Francesca Gavaruzzi $^{2} \cdot$ Giancarlo Ceccarelli $^{2} \cdot$ Cristian Borrazzo $^{2} \cdot$ Alessandra Oliva $^{2}$.

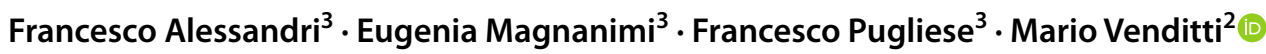

Received: 25 April 2021 / Accepted: 15 June 2021 / Published online: 27 June 2021

(c) The Author(s) 2021

\begin{abstract}
Objectives Superinfections in patients hospitalized in intensive care unit (ICU) are an important and challenging complication, also in COVID-19. However, no definitive data are available about the role of multidrug-resistant Acinetobacter baumannii (MDR-AB) in COVID-19.

Methods This was a single-center, cross-sectional study including patients with MDR-AB infections admitted to ICU with or without COVID-19, between January 2019 and January 2021. The primary objective of the study was to evaluate risk factor for MDR-AB infections in ICU patients hospitalized for COVID-19 or other etiology. The secondary endpoints were 30-days mortality in all study population and risk factors associated with development of bloodstream infection (BSI).

Results During the study period 32 adults with COVID-19 were enrolled and compared with 115 patients admitted in the same ICU for other reasons. We observed a total of 114 deaths, with a survival rate of 29.3\%: $18.8 \%$ in COVID-19 and $32.2 \%$ in control group. Relative risk for MDR-AB infection in COVID-19 showed that serum lactate levels mmol/l $>2$, Acinetobacter baumannii colonization, BSI and steroid therapy were observed more frequently in COVID-19 patients. Cox regression analysis showed that serum lactate levels $>2 \mathrm{mmol} / \mathrm{l}$, Acinetobacter baumannii colonization, BSI, and steroid therapy were associated with 30-days mortality. Finally, patients with COVID-19, white blood cells count $>11,000 \mathrm{~mm}^{3}$, serum lactate levels $>2 \mathrm{mmol} / \mathrm{l}$, infections at time of ICU admission, Acinetobacter baumannii colonization, and steroid therapy were independently associated with development of BSI.

Conclusions Our data highlight the impact of BSI on outcome, the role of Acinetobacter baumannii colonization and the use of steroids on the risk to develop MDR-AB infections also during COVID-19.
\end{abstract}

Keywords Acinetobacter baumannii $\cdot$ COVID-19 $\cdot$ Bacteraemia $\cdot$ Colonization $\cdot$ Steroids

\section{Introduction}

Since the end of 2019 the Coronavirus Disease 2019 (COVID-19), caused by severe acute respiratory syndrome coronavirus 2 (SARS-CoV-2), has spread globally affecting

Mario Venditti

mario.venditti@uniroma1.it

1 Infectious and Tropical Diseases Unit, Department of Medical and Surgical Sciences, "Magna Graecia" University of Catanzaro, Catanzaro, Italy

2 Department of Public Health and Infectious Diseases, "Sapienza" University of Rome, Viale dell'Università 37, 00161 Rome, Italy

3 Department of Anesthesia and Critical Care Medicine, "Sapienza" University of Rome, Rome, Italy people worldwide [1, 2]. Patients with severe COVID-19 require intensive care unit (ICU) admission for acute respiratory failure and over $10 \%$ need noninvasive and invasive mechanical ventilation [3, 4]. Acute respiratory distress syndrome (ARDS) severity and ventilation management determine a negative outcome and a 90-days mortality of 31\% [2].

The data about superinfections complicating COVID-19 are scant, and a significant proportion of these patients are treated with empiric broad spectrum antibiotic therapy that increase the risk to develop infections caused by multidrug-resistant (MDR) pathogens [5, 6]. Finally, the use of drugs targeting cytokines, such as IL- 1 and IL- 6 , might also increase the risk of superinfections in patients with COVID19 [7].

Infections caused by MDR Acinetobacter baumannii (MDR-AB) represent a major problem in patients admitted 
to the intensive care unit (ICU) $[8,9]$. Inappropriate therapy and limited therapeutic options are responsible for negative impact on outcome and this infection is associated with high mortality rates, especially in ICU patients $[10,11]$.

The aim of our study was to evaluate the impact of MDR$\mathrm{AB}$ infections on outcome of patients with COVID-19 requiring ICU admission, comparing with non-COVID-19 patients with MDR-AB infections hospitalized in the same ward. We evaluated risk factor for acquisition of MDR-AB infections in ICU patients hospitalized for COVID-19 or other etiology, 30-days mortality in all study population, and risk factors associated with development of bloodstream infection (BSI).

\section{Methods}

\section{Study design and patient selection}

This was a single-center, cross-sectional study including patients with MDR-AB infections consecutively admitted to the tertiary care Policlinico Umberto I, Sapienza University of Rome, Italy, between January 2019 and January 2021. We compare patients divided in two groups: patients with and without COVID-19 admitted to ICU. Inclusion criteria for all patients were: (1) age $\geq 18$ years; (2) clinical signs and symptoms consistent with infection; (3) documented MDR$A B$ etiology. All patients were managed by the same team of physicians and all antimicrobial therapies were selected according to clinical judgment by infectious disease specialists. The prospective nature of the study was based on the consecutive enrollment of patients. However, all complete data were afterwards retrospectively extracted, and the local Ethics Committee waived the need for informed consent. The study was conducted according to the principles stated in the Declaration of Helsinki.

Patients' data were collected from medical charts and from hospital computerized databases. The following information were reviewed: demographics; clinical, and laboratory findings; comorbid conditions and the age-adjusted Charlson Comorbidity Index; microbiological data; duration of ICU and hospital stay; any infection during hospitalization; duration of antibiotic therapy, and use of steroid therapy; procedures (e.g., mechanical ventilation, continuous renal replacement therapy [CRRT]), extracorporeal membrane oxygenation [ECMO], carried out during hospitalization; the simplified acute physiology score (SAPS II); sequential organ failure assessment (SOFA) and quick (q)-SOFA, serum lactate levels mmol $>2$ at time of infection; anamnestic MDR-AB colonization or infection during hospitalization; source of infection and its adequate control; development of bacteremias and septic shock; 30-days mortality.

\section{Definitions}

Septic shock was defined according to international definitions [12].

The severity of clinical conditions was determined using SAPS II, SOFA, and qSOFA scores calculated at the time of infection onset.

The length of hospital and ICU stay were calculated as the number of days from the date of admission to the date of discharge or death. Adequate control of source of infection was defined as the removal of any preexisting contaminated CVC as well as the drainage of intra-abdominal abscesses or other fluid collections have been performed within $24 \mathrm{~h}$ after the onset of infection. The timing of CVC removal was based on the medical record review, and was confirmed by review of patient radiographs.

MDR-AB infections were classified in the following categories: ventilator-associated pneumonia (VAP), hospital-acquired pneumonia (HAP), urinary tract infection (UTI), and BSI. Acinetobacter baumannii colonization was weekly evaluated with an active surveillance in hospitalized patients.

Inclusion criteria for patients with COVID-19 were: (1) laboratory confirmed SARS-CoV-2 infection with an RT-PCR test on a nasopharyngeal swab; (2) uni- or bilateral interstitial infiltrates confirmed by $\mathrm{CT}$ scan or chest $\mathrm{X}$ ray; (3) presence of acute hypoxemic respiratory failure requiring mechanical ventilation.

All of these clinically indicated infections were categorized as co-infections or superinfections. If diagnosis was at the time of or within the first $24 \mathrm{~h}$ of COVID-19 hospital admission, these infections were defined as communityacquired co-infections. If diagnosis occurred $\geq 48 \mathrm{~h}$ after admission for COVID-19, these infections were defined as hospital-acquired superinfections.

\section{MDR-AB definition}

Identification of MDR-AB strains was based accordingly with local laboratory techniques. The Vitek 2 automated system (bioMérieux, Marcy l'Etoile, France) was used for isolate identification and antimicrobial susceptibility testing. Minimum inhibitory concentrations (MICs) were established according to the European Committee on Antimicrobial Susceptibility Testing (EU- CAST) breakpoints [13]. Isolated strains were classified as multidrug resistant (MDR), extensively drug resistant (XDR), and pandrug resistant (PDR) [14]. 


\section{Primary endpoint and statistical analysis}

The primary objective of the study was to evaluate risk factor for MDR-AB infections in ICU patients hospitalized for COVID-19 or other etiology. The secondary endpoints were 30-days mortality in all study population and risk factors associated with development of BSI.

All data were analyzed using Statistical Package for Social Science (SPSS) version 20 or Microsoft Excel (Office 2018). Description of mean \pm standard deviation (SD), simple frequencies (n), proportions (or percentages), and rates of the given data on each variable has been calculated. The univariate analysis was used to compare patients divided in two groups: MDR-AB infection in patient with COVID19 vs No COVID-19. T test was conducted for continuous variables and chi-square for categorical variables. The odds ratio (OR) and $95 \%$ confidence intervals (CI) were used to quantify the strength of the association between covariates and dependent variable. We have done a standard survival analysis, tracing participants affected, or not by COVID19 from entry into the clinic to the discharge or death at 30 days. The event-free survival in follow-up was depicted graphically by Kaplan-Meier's survival curve, including the confounding factors with fixed baseline covariates. A $p$ value of less than 0.05 was considered statistically significant.

\section{Results}

During the study period, 32 adults with COVID-19 and superinfection caused by MDR-AB were prospectively enrolled. This cohort of patients was compared with 115 patients with MDR-AB infection admitted in the same ICU for other etiologies: respiratory failure (29\%), septic shock
(26\%), trauma (20\%), stroke (15\%), cardiac/hemorrhagic shock/postsurgery (10\%). Overall, 147 patients were evaluated in the final analysis. Sites of MDR-AB infection in study population are reported in Fig. 1.

Table 1 shows univariate analysis comparing clinical characteristics of patients affected or not by COVID-19 with documented MDR-AB infection. Differences between COVID-19 and other patients were reported for previous hospitalization ( $16 \%$ vs. $39 \%, p<0.015)$, chronic kidney disease ( $3 \%$ vs. $19 \%, p<0.001)$, COPD (9\% vs. $32 \%$, $p<0.009)$, chronic corticosteroid therapy (0\% vs. $27 \%$, $p<0.001$ ), and previous Acinetobacter baumannii colonization or infection $(0 \%$ vs. $15 \%, p<0.020)$. No differences were observed between COVID-19 and other patients related to the median age, Charlson Comorbidity Index, length of hospitalization, and ICU stay, SAPS II at time of admission, procedures (e.g., mechanical ventilation), clinical and laboratory findings at time of infection, duration of antibiotic therapy, development of septic shock, 30-days mortality.

Relative risk associated with COVID-19 vs. nonCOVID-19 etiology was reported in Table 2: previous hospitalization (RR 0.4; CI 95\% 0.2-0.9, $p=0.031$ ), COPD (RR 0.3, CI 95\% 0.1-0.9, $p=0.029$ ), chronic corticosteroid therapy (RR 0.1, CI 95\% 0.0-0.9, $p=0.041$ ) and infection at time of ICU admission (RR 0.1, CI 95\% 0.0-0.9, $p=0.001$ ) were factors associated with non-COVID-19 etiology. Conversely, serum lactate levels $>2 \mathrm{mmol} / \mathrm{l}$ at time of infection (RR 1.8, CI 95\% 1.3-2.5, $p=0.001$ ), Acinetobacter baumannii colonization (RR 7.9, CI 95\% 4.0-15.7, $p<0.001$ ), BSI (RR 6.5, CI 95\% 3.2-13.3, $p<0.001$ ), and steroid therapy (RR 18.4, CI 95\% 7.6-44.1, $p<0.001$ ) were observed more frequently in COVID-19 patients.

Cox regression analysis of factors associated with 30-days mortality (see Table 3) showed that serum lactate
Fig. 1 Sites of MDR-AB infection in COVID-19 (gray line) or non-COVID-19 (black line). $M D R-A B$ multidrug-resistant Acinetobacter baumannii, BSI bloodstream infection, VAP ventilator-associated pneumonia, UTI urinary tract infection, CVC central venous catheter, $V A T$ ventilator-associated tracheobronchitis

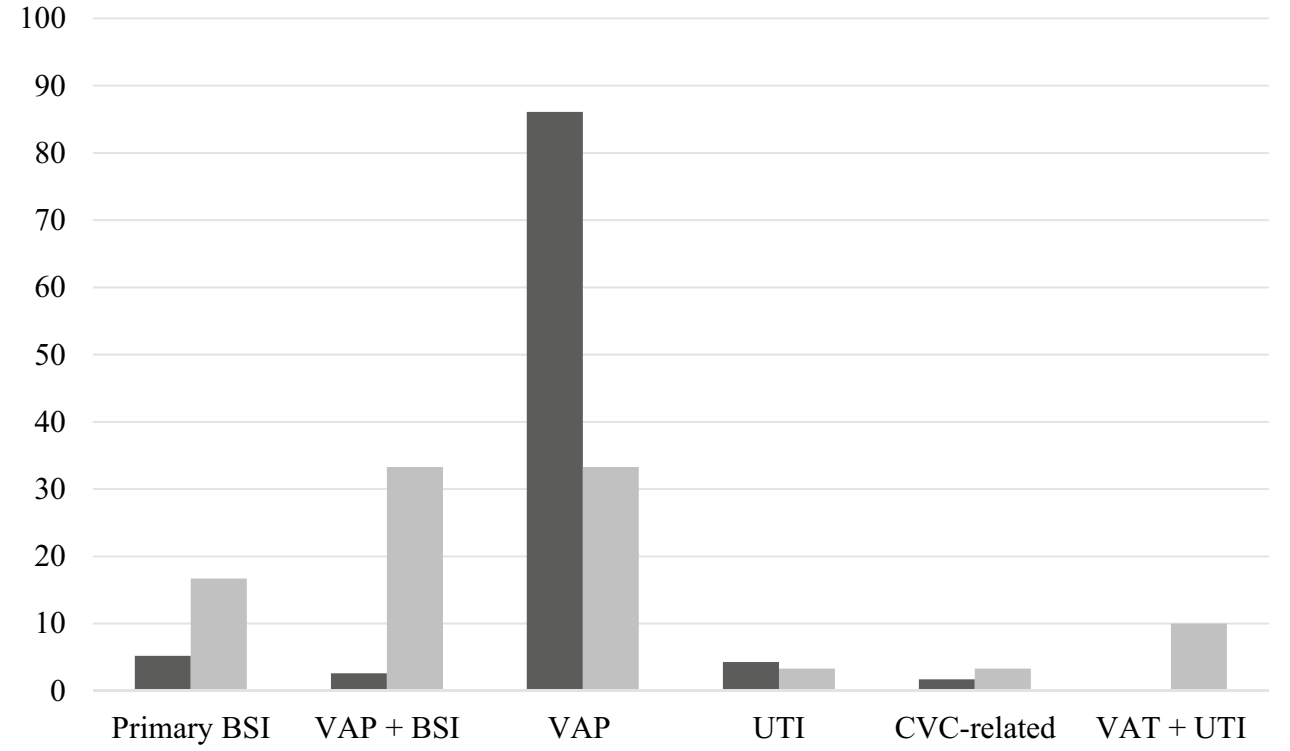


Table 1 Univariate analysis about clinical characteristics and outcome of patients with MDR-AB infections affected or not by COVID-19

\begin{tabular}{|c|c|c|c|}
\hline Variables & $\begin{array}{l}\text { COVID-19 } \\
n=32(\%)\end{array}$ & $\begin{array}{l}\text { Non-COVID-19 } \\
n=115(\%)\end{array}$ & $p$ value \\
\hline \multicolumn{4}{|l|}{ Anamnestic factors and comorbidities } \\
\hline Male sex & $21(66 \%)$ & $73(63 \%)$ & 0.543 \\
\hline Age, mean $\pm \mathrm{SD}$ (years) & $62.50 \pm 10.99$ & $62.59 \pm 11.31$ & 0.969 \\
\hline Previous hospitalization (90 days) & $5(16 \%)$ & $45(39 \%)$ & 0.015 \\
\hline Previous ICU admission (90 days) & $3(9 \%)$ & $22(19 \%)$ & 0.132 \\
\hline$>2$ comorbidities & $11(34 \%)$ & $41(36 \%)$ & 0.895 \\
\hline Cardiovascular disease & $17(53 \%)$ & $62(54 \%)$ & 0.938 \\
\hline Heart failure & $3(9 \%)$ & $9(8 \%)$ & 0.791 \\
\hline Charlson Comorbidity Index, mean \pm SD & $2.59 \pm 1.81$ & $2.79 \pm 1.78$ & 0.605 \\
\hline Diabetes & $4(13 \%)$ & $12(10 \%)$ & 0.756 \\
\hline Chronic kidney disease & $1(3 \%)$ & $22(19 \%)$ & 0.001 \\
\hline Chronic liver disease & $1(3 \%)$ & $8(7 \%)$ & 0.333 \\
\hline Neurologic disease & $3(9 \%)$ & $19(17 \%)$ & 0.267 \\
\hline Vasculitis & $1(3 \%)$ & $9(8 \%)$ & 0.245 \\
\hline COPD & $3(9 \%)$ & $37(32 \%)$ & 0.009 \\
\hline Solid tumor & $2(6 \%)$ & $9(8 \%)$ & 0.755 \\
\hline Hematological malignancies & $1(3 \%)$ & $8(7 \%)$ & 0.333 \\
\hline Chronic corticosteroid therapy & 0 & $31(27 \%)$ & 0.001 \\
\hline Previous Acinetobacter baumannii infection (30 days) & 0 & $17(15 \%)$ & $\mathbf{0 . 0 2 0}$ \\
\hline Previous endoscopy procedure (30 days) & $3(9 \%)$ & $16(14 \%)$ & 0.464 \\
\hline Intravascular devices & $5(16 \%)$ & $17(15 \%)$ & 0.909 \\
\hline Previous antibiotic therapy (30 days) & $9(28 \%)$ & $45(39 \%)$ & 0.241 \\
\hline \multicolumn{4}{|l|}{ Clinical features } \\
\hline SAPS II at time of admission, mean \pm SD & $33.75 \pm 15.57$ & $37.10 \pm 17.75$ & 0.296 \\
\hline GCS at the time of admission, mean \pm SD & $14.65 \pm 0.00$ & $15.00 \pm 0.00$ & 0.330 \\
\hline $\mathrm{PaO}_{2} / \mathrm{FiO}_{2}<250$ & $26(81 \%)$ & $88(77 \%)$ & 0.560 \\
\hline $\mathrm{WBC}$, mean $\pm \mathrm{SD}$ & $10,937.81 \pm 8794.10$ & $13,411.83 \pm 12,375.35$ & 0.209 \\
\hline Surgery & $2(6 \%)$ & $9(8 \%)$ & 0.755 \\
\hline PLTs, mean \pm SD & $254,656.25 \pm 101,504.71$ & $246,200.00 \pm 88,537.26$ & 0.679 \\
\hline SOFA at the time of admission, mean \pm SD & $5.31 \pm 2.95$ & $4.92 \pm 2.55$ & 0.487 \\
\hline Quick SOFA at the time of admission, mean \pm SD & $0.72 \pm 0.70$ & $0.83 \pm 0.81$ & 0.454 \\
\hline CRRT & $13(41 \%)$ & $46(40 \%)$ & 0.949 \\
\hline ECMO & $4(13 \%)$ & $15(13 \%)$ & 1.000 \\
\hline Surgery source control & $3(9 \%)$ & $20(17 \%)$ & 0.267 \\
\hline Septic shock & $8(25 \%)$ & $43(37 \%)$ & 0.208 \\
\hline SOFA at time of infection onset, mean \pm SD & $8.31 \pm 4.29$ & $7.48 \pm 3.76$ & 0.312 \\
\hline Quick SOFA at time of infection onset, mean \pm SD & $1.25 \pm 0.90$ & $1.28 \pm 0.93$ & 0.875 \\
\hline $\mathrm{PCT}$ at time of infection onset, mean $\pm \mathrm{SD}$ & $4.01 \pm 6.33$ & $3.73 \pm 5.23$ & 0.825 \\
\hline Serum lactate levels $>2 \mathrm{mmol} / 1$ & $21(66 \%)$ & $42(37 \%)$ & 0.003 \\
\hline MDR colonization at the time of ICU admission & $1(3 \%)$ & $16(14 \%)$ & 0.068 \\
\hline Infections at time of ICU admission & $2(6 \%)$ & $70(61 \%)$ & $<0.001$ \\
\hline Acinetobacter baumannii colonization & $20(63 \%)$ & $9(8 \%)$ & $<0.001$ \\
\hline Time from colonization to MDR-AB infection, mean \pm SD (days) & $10.21 \pm 9.85$ & $11.82 \pm 9.2$ & 0.89 \\
\hline Bloodstream infection & $18(56 \%)$ & $9(8 \%)$ & $<0.001$ \\
\hline \multicolumn{4}{|l|}{ Outcomes and therapy } \\
\hline Steroid therapy & $28(88 \%)$ & $5(4 \%)$ & $<0.001$ \\
\hline Total duration of antibiotic therapy, mean \pm SD & $25.56 \pm 12.66$ & $25.35 \pm 14.87$ & 0.936 \\
\hline Transfer in ICU & $31(97 \%)$ & $115(100 \%)$ & 0.325 \\
\hline Length of ICU stay, mean \pm SD (days) & $22.22 \pm 9.65$ & $22.23 \pm 9.53$ & 0.997 \\
\hline Length of hospitalization, mean $\pm \mathrm{SD}$ (days) & $30.41 \pm 13.56$ & $29.10 \pm 10.59$ & 0.610 \\
\hline Mortality at 30 days & $26(81 \%)$ & $78(68 \%)$ & 0.154 \\
\hline
\end{tabular}


Table 1 (continued)

Statistically significant $p$-values are in bold

$M D R-A B$ multidrug-resistant Acinetobacter baumannii, $S D$ standard deviation, $I C U$ intensive care unit, $C O P D$ chronic obstructive pulmonary disease, $S A P S$ simplified acute physiology score, GCS Glasgow coma score, WBC white blood cell, PLT platelets, SOFA sequential organ failure assessment, PICC peripherally-inserted central catheter, $C V C$ central venous catheter, $C R R T$ continuous renal replacement therapy, ECMO extracorporeal membrane oxygenation, $P C T$ procalcitonin, $C R P$ C-reactive protein

Table 2 Relative risk* associated or not with MDR-AB infection in patients affected or not by COVID-19

\begin{tabular}{lrrr}
\hline Variables & RR & CI 95\% & $p$ value \\
\hline Previous hospitalization (90 days) & 0.4 & $0.2-0.9$ & 0.031 \\
COPD & 0.3 & $0.1-0.9$ & 0.029 \\
Chronic steroid therapy & 0.1 & $0.0-0.9$ & 0.041 \\
Infection at time of ICU admission & 0.1 & $0.0-0.4$ & 0.001 \\
Serum lactate levels > 2 mmol/1 & 1.8 & $1.3-2.5$ & 0.001 \\
Acinetobacter baumannii colonization & 7.9 & $4.0-15.7$ & $<0.001$ \\
Bloodstream infection & 6.5 & $3.2-13.3$ & $<0.001$ \\
Steroid therapy & 18.4 & $7.6-44.1$ & $<0.001$ \\
\hline
\end{tabular}

$R R$ relative risk, $C I$ confidence interval, $C O P D$ chronic obstructive pulmonary disease, $I C U$ intensive care unit

$* \mathrm{RR}<1$ is associated with non-COVID-19 etiology; $>1$ with COVID-19

Table 3 Logistic regression analysis about risk factors associated with 30-days mortality

\begin{tabular}{lrlr}
\hline Variables & OR & CI 95\% & $p$ value \\
\hline Serum lactate levels $>2$ mmol/1 & 4.9 & $2.1-11.3$ & $<0.001$ \\
Acinetobacter baumannii colonization & 17.1 & $5.5-53.3$ & $<0.001$ \\
Bloodstream infection & 13.6 & $4.8-38.2$ & $<0.001$ \\
Steroid therapy & 46.9 & $13.9-157.5$ & $<0.001$ \\
\hline
\end{tabular}

$O R$ odds ratio, $C I$ confidence interval

levels $>2 \mathrm{mmol} / \mathrm{l}$ at time of infection (OR 4.9; CI 95\% 2.1-11.3, $p<0.001)$, Acinetobacter baumannii colonization (OR 17.1, CI 95\% 5.5-53.3, $p<0.001$ ), BSI (OR 13.6; CI $95 \% 4.8-38.2, p<0.001$ ) and steroid therapy (OR 46.9; CI $95 \% 13.9-157.3, p<0.001)$ were associated with 30-days mortality.

The Kaplan-Meier curves for 30-days survival of overall patients with MDR-AB infections (COVID-19 or nonCOVID-19 etiology) is reported in Fig. 2. We observed a total of 114 deaths, with a survival rate of $29.3 \%$. Comparing the two groups, we observed a different survival rate: $18.8 \%$ (COVID-19) and $32.2 \%$ (other patients).

Table 4 shows univariate analysis comparing patients developing or not BSI caused by MDR-AB. COVID-19 etiology $(67 \%$ vs. $12 \%, p<0.001)$, serum lactate levels $>2 \mathrm{mmol} / \mathrm{l}$ at time of infection ( $63 \%$ vs. $38 \%, p=0.018)$, Acinetobacter baumannii colonization (44\% vs. $14 \%$, $p=0.006)$ and a steroid therapy during the hospitalization ( $63 \%$ vs. $13 \%, p<0.001)$ were more frequently reported in patients developing MDR-AB BSI.

Finally, multivariate analysis about risk factors associated with development of BSI (see Table 5) showed that patients with COVID-19 (OR 15.1, CI 95\% 3.7-40.1; $p<0.001$ ), white blood cells count $>11,000 \mathrm{~mm}^{3}$ (OR 5.2, CI $95 \%$ $2.1-11.5 ; p<0.001$ ), serum lactate levels $>2 \mathrm{mmol} / \mathrm{l}$ (OR 2.7; CI 95\% 1.2-6.4, $p=0.022$ ), infections at time of ICU admission (OR 0.4, CI 95\% 0.2-1.0, $p=0.030$ ), Acinetobacter baumannii colonization (OR 4.8, CI 95\% 1.9-12.1, $p<0.001$ ), and steroid therapy during hospitalization (OR 8.8 , CI 95\% 3.5-22.1, $p<0.001$ ) were factors independently associated with development of BSI.

\section{Discussion}

To our knowledge, this is the largest experience about risk factors and outcomes of MDR-AB infections in patients affected or not by COVID-19 in ICU. Our study confirms that bacterial superinfections may complicate the hospital course of patients with COVID-19, and we identified peculiar characteristics of COVID-19 patients developing these difficult-to-treat infections. Our data showed that serum lactate levels $>2 \mathrm{mmol} / \mathrm{l}$ at time of infection, Acinetobacter baumannii colonization, development of a BSI, and steroid therapy were the most important factors associated with MDR-AB infection in COVID-19 patients and resulted as important determinants of 30-day mortality also in all study population.

Moreover, COVID-19 etiology, white blood cells count $>11,000 \mathrm{~mm}^{3}$, serum lactate levels $>2 \mathrm{mmol} / 1$, infection at time of ICU admission, Acinetobacter baumannii colonization, and steroid therapy during hospitalization were associated with higher risk of BSI development. In this study, our data showed as MDR-AB BSI remain an important ICU-acquired infection [8, 9, 11-15].

Of interest, our experience highlighted the importance of superinfections caused by Gram-negative strains in ICU, including COVID-19 patients. Of importance, a rapid spread of MDR gram-negative bacteria among patients in dedicated coronavirus disease care units was recently observed [16]. In a recent meta-analysis, $19 \%$ of patients with COVID-19 showed co-infections and $24 \%$ superinfections; the presence 
Fig. 2 Kaplan-Meier curves for 30-days survival in patients affected (green line) or not (blue line) by COVID-19

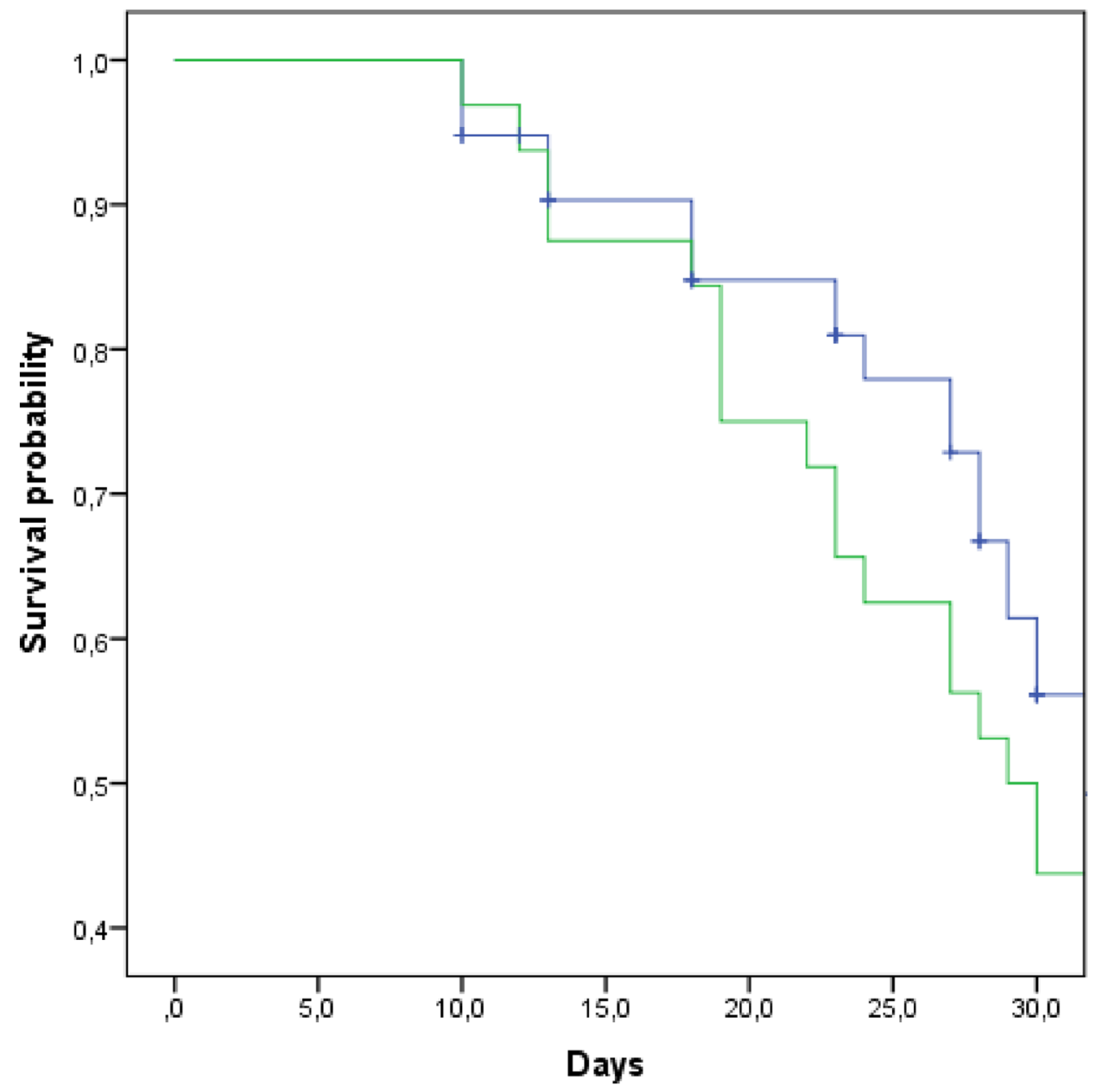

of either co-infection or superinfection was associated with poor outcomes, including increased mortality [17].

First of all, this data reflect local epidemiology characterized by a high prevalence of MDR Gram-negatives. The data from literature suggest that COVID-19 was associated with a less effective implementation of infection control procedures for several reasons [18]. As a matter of fact, healthcare workers (HCWs) experimented important difficulties to apply standard precautions, and to wear the same equipment for a long time; moreover, HCWs mainly focused on self-protection rather than on cross-transmission of bacteria in the wards. Finally, overcrowded wards, shortages of professionals with appropriate training in infection control procedures, and possible decreased laboratory ability to detect MDR carriage are potentially considered risk factors of MDR spread after the COVID-19 outbreak [19, 20]. Then, it will be crucial to continue monitoring rates of MDR infections and implementing measures of infection control and antimicrobial stewardship [21-23].

Of note, Acinetobacter baumannii intestinal colonization resulted as an independent predictor of infection in COVID-19 patients. As a matter of fact, the association between rectal carriage by carbapenem-resistant pathogens and development of infection is reported as an important predictor of infection, especially in ICU patients [24]. There are many important observations about the role of the gut microbiota during SARS-CoV-2 infection. The gut microbiota of COVID-19 patients is characterized by enrichment of opportunistic pathogens and alterations in gut cells also in the absence of gastrointestinal manifestations [19, 25, 26]. Of interest, these alterations were observed after hospitalization; however, the administration of broad-spectrum antibiotics is the major determinant for intestinal colonization by MDR pathogens.

Our data showed that MDR-AB BSI remains an important ICU-acquired infection, associated with higher mortality. In our interpretation, sepsis and septic shock determine a lethal cascade of events that is unlikely to be interrupted even by an appropriate initial antimicrobial treatment. In addition, most of our patients were severely ill and would probably have been unable to survive their infections independently of the administration of an adequate initial antimicrobial treatment. The data about the high rate of unfavorable outcome in patient with MDR-AB BSI were previously reported and 
Table 4 Univariate analysis comparing patients developing or not bloodstream infection

\begin{tabular}{|c|c|c|c|}
\hline Variables & $\begin{array}{l}\text { No bloodstream infection } \\
n=120(\%)\end{array}$ & $\begin{array}{l}\text { Bloodstream infection } \\
n=27(\%)\end{array}$ & $p$ value \\
\hline \multicolumn{4}{|l|}{ Anamnestic factors and comorbidities } \\
\hline COVID-19 & $14(12 \%)$ & $18(67 \%)$ & $<0.001$ \\
\hline Male sex & $77(64 \%)$ & $17(63 \%)$ & 0.922 \\
\hline Age, mean $\pm \mathrm{SD}$ (years) & $61.84 \pm 11.55$ & $65.81 \pm 9.86$ & 0.074 \\
\hline Previous hospitalization (90 days) & $42(35 \%)$ & $8(30 \%)$ & 0.593 \\
\hline Previous ICU admission (90 days) & $22(18 \%)$ & $3(11 \%)$ & 0.315 \\
\hline$>2$ comorbidities & $43(36 \%)$ & $9(33 \%)$ & 0.808 \\
\hline Cardiovascular disease & $67(56 \%)$ & $12(44 \%)$ & 0.260 \\
\hline Heart failure & $10(8 \%)$ & $2(7 \%)$ & 0.872 \\
\hline Charlson Comorbidity Index mean \pm SD & $2.73 \pm 1.78$ & $2.85 \pm 1.97$ & 0.761 \\
\hline Diabetes & $13(11 \%)$ & $3(11 \%)$ & 1.000 \\
\hline Chronic kidney disease & $18(15 \%)$ & $5(19 \%)$ & 0.674 \\
\hline Chronic liver disease & $9(8 \%)$ & $0(0 \%)$ & 0.129 \\
\hline Neurologic disease & $17(14 \%)$ & $5(19 \%)$ & 0.602 \\
\hline Vasculitis & $8(7 \%)$ & $2(7 \%)$ & 1.000 \\
\hline COPD & $34(28 \%)$ & $6(22 \%)$ & 0.508 \\
\hline Solid tumor & $10(8 \%)$ & $1(4 \%)$ & 0.307 \\
\hline Hematological malignancies & $9(8 \%)$ & 0 & 0.129 \\
\hline Chronic corticosteroid therapy & $27(23 \%)$ & $4(15 \%)$ & 0.339 \\
\hline Previous Acinetobacter baumannii infection (30 days) & $16(13 \%)$ & $1(4 \%)$ & 0.051 \\
\hline Previous endoscopy procedure (30 days) & $18(15 \%)$ & $1(4 \%)$ & 0.157 \\
\hline Intravascular devices & $16(13 \%)$ & $6(22 \%)$ & 0.316 \\
\hline Previous antibiotic therapy (30 days) & $47(39 \%)$ & $7(26 \%)$ & 0.179 \\
\hline \multicolumn{4}{|l|}{ Clinical features } \\
\hline SAPS II at time of admission, mean \pm SD & $37.01 \pm 17.69$ & $33.56 \pm 15.24$ & 0.308 \\
\hline GCS at the time of admission, mean \pm SD & $15.00 \pm 0.00$ & $14.63 \pm 1.61$ & 0.331 \\
\hline $\mathrm{PaO} 2 / \mathrm{FiO} 2<250$ & $95(79 \%)$ & $19(70 \%)$ & 0.207 \\
\hline $\mathrm{WBC}$ mean $\pm \mathrm{SD}$ & $13,707.75 \pm 12,658.93$ & $9164.44 \pm 4563.52$ & 0.002 \\
\hline Surgery & $10(8 \%)$ & $1(4 \%)$ & 0.307 \\
\hline $\mathrm{PTL}$ mean $\pm \mathrm{SD}$ & $248,166.67 \pm 88,895.02$ & $247,481.48 \pm 106,697.40$ & 0.975 \\
\hline SOFA at the time of admission, mean $\pm \mathrm{SD}$ & $5.02 \pm 2.59$ & $4.96 \pm 2.79$ & 0.928 \\
\hline Quick SOFA at the time of admission, mean $\pm \mathrm{SD}$ & $0.83 \pm 0.81$ & $0.67 \pm 0.62$ & 0.241 \\
\hline CRRT & $45(37.5)$ & $14(51.8)$ & 0.06 \\
\hline ECMO & $18(15 \%)$ & $1(4 \%)$ & 0.157 \\
\hline Surgery source control & $22(18 \%)$ & $1(4 \%)$ & 0.069 \\
\hline Septic shock & $40(33.3)$ & $11(40.7)$ & 0.384 \\
\hline SOFA at time of infection onset, mean \pm SD & $7.44 \pm 3.72$ & $8.63 \pm 4.33$ & 0.196 \\
\hline qSOFA at time of infection onset, mean \pm SD & $1.24 \pm 0.92$ & $1.41 \pm 0.93$ & 0.407 \\
\hline PCT at time of infection onset, mean \pm SD & $3.75 \pm 5.52$ & $3.97 \pm 5.18$ & 0.847 \\
\hline Serum lactate levels $>2 \mathrm{mmol} / \mathrm{l}$ & $46(38 \%)$ & $17(63 \%)$ & 0.018 \\
\hline MDR colonization at the time of ICU admission & $15(13 \%)$ & $2(7 \%)$ & 0.386 \\
\hline Infection at time of ICU admission & $64(53 \%)$ & $8(30 \%)$ & 0.031 \\
\hline Acinetobacter baumannii colonization & $17(14 \%)$ & $12(44 \%)$ & 0.006 \\
\hline Time from colonization to MDR-AB infection, mean \pm SD (days) & $13.34 \pm 11.81$ & $10.91 \pm 8.2$ & 0.03 \\
\hline \multicolumn{4}{|l|}{ Outcomes and therapy } \\
\hline Steroid therapy & $16(13 \%)$ & $17(63 \%)$ & $<0.001$ \\
\hline Total duration of antibiotic therapy, mean \pm SD & $25.83 \pm 15.19$ & $23.44 \pm 10.48$ & 0.333 \\
\hline Transfer in ICU & $120(100 \%)$ & $26(96 \%)$ & 0.327 \\
\hline Length of ICU stay, mean \pm SD (days) & $21.75 \pm 9.17$ & $24.33 \pm 11.51$ & 0.283 \\
\hline Length of hospitalization, mean $\pm \mathrm{SD}$ (days) & $29.46 \pm 11.20$ & $29.07 \pm 11.25$ & 0.873 \\
\hline Mortality at 30 days & $84(70 \%)$ & $20(74 \%)$ & 0.673 \\
\hline
\end{tabular}


Table 4 (continued)

Statistically significant $p$-values are in bold

$M D R-A B$ multidrug-resistant Acinetobacter baumannii, $S D$ standard deviation, $I C U$ intensive care unit, $C O P D$ chronic obstructive pulmonary disease, $S A P S$ simplified acute physiology score, GCS Glasgow coma score, WBC white blood cell, PLT platelets, SOFA sequential organ failure assessment, PICC peripherally-inserted central catheter, $C V C$ central venous catheter, CRRT continuous renal replacement therapy, ECMO extracorporeal membrane oxygenation, $P C T$ procalcitonin, $C R P$ C-reactive protein

Table 5 Multivariate analysis about risk factors associated with development of bloodstream infection

\begin{tabular}{lrlr}
\hline Variables & OR & CI 95\% & $p$ value \\
\hline Severe COVID-19 & 15.1 & $3.7-40.1$ & $<0.001$ \\
WBC $>11,000 \mathrm{~mm}^{3}$ & 5.2 & $2.1-11.5$ & $<0.001$ \\
Serum lactate levels $>2$ mmol/1 & 2.7 & $1.2-6.4$ & 0.022 \\
Infections at time of ICU admission & 0.4 & $0.2-1$ & 0.030 \\
Acinetobacter baumannii colonization & 4.8 & $1.9-12.1$ & $<0.001$ \\
Steroid therapy & 8.8 & $3.5-22.1$ & $<0.001$ \\
\hline
\end{tabular}

$O R$ odds ratio, $C I$ confidence interval, $W B C$ white blood cell, $I C U$ intensive care unit

discussed [8, 9, 11]. Finally, MDR-AB can be considered as a marker of the severity for the underlying diseases.

A peculiar aspect of COVID-19 patients, especially during the "second wave", was the widely use of steroids at high dosages. The use of dexamethasone resulted in lower 28-day mortality, especially in patients receiving invasive mechanical ventilation [27, 28]. However, the prolonged use of high doses of steroids could be associated with the wellknown immunomodulant effects of these drugs [29], but the association between steroid treatment and MDR infections deserves further comments. We can hypothesize that patients who received steroids survived longer and were, therefore, more likely to develop an MDR infection during ICU stay. However, in COVID-19 patients with a prolonged ICU stay the use of steroids and immunomodulant drugs may increase the risk of superinfections and should be used with caution $[30,31]$.

Our study has some limitations. First, it is a singlecenter study conducted in a setting with a high prevalence of MDR pathogens. Second, the sample size is relatively small, and the CIs of some significant predictors are quite broad. Third, we did not perform a multilocus sequence typing of the strains to understand if we observed an outbreak of infection. Finally, the impact of some therapies, including immunomodulant drugs, and of empiric and definitive antibiotic regimens for treatment of MDR-AB infections were not definitively assessed in the final analysis.

In conclusion, we reported a single-center experience about MDR-AB infection in COVID-19 patients, comparing those with ICU patients hospitalized for other etiologies. Our data highlight the impact of BSI on outcome, the role of Acinetobacter baumannii colonization and the use of steroids on the risk to develop MDR-AB infections also during COVID-19. Antimicrobial stewardship programs are mandatory in this population $[32,33]$.

Funding Open access funding provided by Università degli Studi di Roma La Sapienza within the CRUI-CARE Agreement. None.

\section{Declarations}

Conflict of interest The authors declare that they have no conflict of interest.

Transparency declarations None to declare.

Open Access This article is licensed under a Creative Commons Attribution 4.0 International License, which permits use, sharing, adaptation, distribution and reproduction in any medium or format, as long as you give appropriate credit to the original author(s) and the source, provide a link to the Creative Commons licence, and indicate if changes were made. The images or other third party material in this article are included in the article's Creative Commons licence, unless indicated otherwise in a credit line to the material. If material is not included in the article's Creative Commons licence and your intended use is not permitted by statutory regulation or exceeds the permitted use, you will need to obtain permission directly from the copyright holder. To view a copy of this licence, visit http://creativecommons.org/licenses/by/4.0/.

\section{References}

1. Serafim RB, Póvoa P, Souza-Dantas V, Kalil AC, Salluh JIF. Clinical course and outcomes of critically ill patients with COVID-19 infection: a systematic review. Clin Microbiol Infect. 2021;27:47-54.

2. Huang C, Soleimani J, Herasevich S, Pinevich Y, Pennington KM, Dong Y, Pickering BW, Barwise AK. Clinical characteristics, treatment, and outcomes of critically Ill patients with COVID19: a scoping review. Mayo Clin Proc. 2021;96:183-202.

3. Zhou F, Yu T, Du R, Fan G, Liu Y, Liu Z, Xiang J, Wang Y, Song B, Gu X, Guan L, Wei Y, Li H, Wu X, Xu J, Tu S, Zhang Y, Chen H, Cao B. Clinical course and risk factors for mortality of adult inpatients with COVID-19 in Wuhan, China: a retrospective cohort study. Lancet. 2020;395:1054-62 (Erratum in: Lancet. 2020;395:1038).

4. Rouzé A, Martin-Loeches I, Povoa P, Makris D, Artigas A, Bouchereau M, Lambiotte F, Metzelard M, Cuchet P, Boulle Geronimi C, Labruyere M, Tamion F, Nyunga M, Luyt CE, Labreuche J, Pouly O, Bardin J, Saade A, Asfar P, Baudel JL, Beurton A, Garot D, Ioannidou I, Kreitmann L, Llitjos JF, Magira E, Mégarbane B, Meguerditchian D, Moglia E, Mekontso-Dessap A, Reignier J, Turpin M, Pierre A, Plantefeve G, Vinsonneau C, Floch PE, Weiss N, Ceccato A, Torres A, Duhamel A, Nseir S, 
coVAPid study Group. Relationship between SARS-CoV-2 infection and the incidence of ventilator-associated lower respiratory tract infections: a European multicenter cohort study. Intensive Care Med. 2021;47:188-98.

5. Lima WG, Brito JCM, da Cruz Nizer WS. Ventilator-associated pneumonia (VAP) caused by carbapenem-resistant Acinetobacter baumannii in patients with COVID-19: two problems, one solution? Med Hypotheses. 2020;144: 110139.

6. Contou D, Claudinon A, Pajot O, Micaëlo M, Longuet Flandre P, Dubert M, Cally R, Logre E, Fraissé M, Mentec H, Plantefève G. Bacterial and viral co-infections in patients with severe SARSCoV-2 pneumonia admitted to a French ICU. Ann Intensive Care. 2020;10:119.

7. Pettit NN, Nguyen CT, Mutlu GM, Wu D, Kimmig L, Pitrak D, Pursell K. Late onset infectious complications and safety of tocilizumab in the management of COVID-19. J Med Virol. 2021;93:1459-64.

8. Russo A, Bassetti M, Ceccarelli G, Carannante N, Losito AR, Bartoletti M, Corcione S, Granata G, Santoro A, Giacobbe DR, Peghin M, Vena A, Amadori F, Segala FV, Giannella M, Di Caprio G, Menichetti F, Del Bono V, Mussini C, Petrosillo N, De Rosa FG, Viale P, Tumbarello M, Tascini C, Viscoli C, Venditti M, ISGRI-SITA (Italian Study Group on Resistant Infections of the Società Italiana Terapia Antinfettiva). Bloodstream infections caused by carbapenem-resistant Acinetobacter baumannii: Clinical features, therapy and outcome from a multicenter study. J Infect. 2019;79:130-8.

9. Russo A, Bassetti M, Bellelli V, Bianchi L, Marincola Cattaneo F, Mazzocchetti S, Paciacconi E, Cottini F, Schiattarella A, Tufaro G, Sabetta F, D'Avino A. Efficacy of a fosfomycin-containing regimen for treatment of severe pneumonia caused by multidrugresistant Acinetobacter baumannii: a prospective, observational study. Infect Dis Ther. 2021;10:187-200.

10. Bassetti M, Righi E, Vena A, Graziano E, Russo A, Peghin M. Risk stratification and treatment of ICU-acquired pneumonia caused by multidrug- resistant/extensively drug-resistant/pandrugresistant bacteria. Curr Opin Crit Care. 2018;24:385-93.

11. Russo A, Giuliano S, Ceccarelli G, Alessandri F, Giordano A, Brunetti G, Venditti M. Comparison of septic shock due to multidrug-resistant Acinetobacter baumannii or Klebsiella pneumoniae carbapenemase-producing $K$. pneumoniae in intensive care unit patients. Antimicrob Agents Chemother. 2018;62:e02562-e2617.

12. Singer M, Deutschman CS, Seymour CW, Shankar- Hari M, Annane D, Bauer M, et al. The third international consensus definitions for sepsis and septic shock (Sepsis-3). JAMA. 2016;315:801-10.

13. European Committee on Antimicrobial Susceptibility Testing (EUCAST). Break- point tables for interpretation of MICs and zone diameters, Växjö, Sweden: European Committee on Antimicrobial Susceptibility Testing; 2021, https://eucast.org/clinical_ breakpoints/. Accessed 31 Mar 2021.

14. Magiorakos AP, Srinivasan A, Carey RB, Carmeli Y, Falagas ME, Giske CG, et al. Multidrug-resistant, extensively drug-resistant and pandrug-resistant bacteria: an international expert proposal for interim standard definitions for acquired resistance. Clin Microbiol Infect. 2012;18:268-81.

15. Wong D, Nielsen TB, Bonomo RA, Pantapalangkoor P, Luna B, Spellberg B. Clinical and pathophysiological overview of acinetobacter infections: a century of challenges. Clin Microbiol Rev. 2017;30:409-47.

16. Patel A, Emerick M, Cabunoc MK, Williams MH, Preas MA, Schrank G, Rabinowitz R, Luethy P, Johnson JK, Leekha S. Rapid spread and control of multidrug-resistant gram-negative bacteria in COVID-19 patient care units. Emerg Infect Dis. 2021;27:1234-7.
17. Musuuza JS, Watson L, Parmasad V, Putman-Buehler N, Christensen L, Safdar N. Prevalence and outcomes of co-infection and superinfection with SARS-CoV-2 and other pathogens: a systematic review and meta-analysis. PLoS ONE. 2021;16: e0251170.

18. d'Ettorre G, Ceccarelli G, Santinelli L, Vassalini P, Innocenti GP, Alessandri F, Koukopoulos AE, Russo A, d'Ettorre G, Tarsitani L. Post-traumatic stress symptoms in healthcare workers dealing with the COVID-19 Pandemic: a systematic review. Int J Environ Res Public Health. 2021;18:601. https://doi.org/10.3390/ijerp h18020601.

19. Falcone M, Tiseo G, Giordano C, Leonildi A, Menichini M, Vecchione A, Pistello M, Guarracino F, Ghiadoni L, Forfori F, Barnini S, Menichetti F, Pisa COVID-19 Study Group. Predictors of hospital-acquired bacterial and fungal superinfections in COVID-19: a prospective observational study. J Antimicrob Chemother. 2021;76:1078-84.

20. Monnet DL, Harbarth S. Will coronavirus disease (COVID19) have an impact on antimicrobial resistance? Euro Surveill. 2020;25:2001886

21. Isler B, Doi Y, Bonomo RA, Paterson DL. New treatment options against carbapenem-resistant Acinetobacter baumannii infections. Antimicrob Agents Chemother. 2018;63:e01110-e1118.

22. Bengoechea JA, Bamford CG. SARS-CoV-2, bacterial co-infections, and AMR: the deadly trio in COVID-19? EMBO Mol Med. 2020;12: e12560.

23. Russo A. Spotlight on new antibiotics for the treatment of pneumonia. Clin Med Insights Circ Respir Pulm Med. 2020;14:1179548420982786.

24. Cano A, Gutiérrez-Gutiérrez B, Machuca I, et al. Risks of infection and mortality among patients colonized with Klebsiella pneumoniae carbapenemase-producing $K$. pneumoniae: validation of scores and proposal for management. Clin Infect Dis. 2018;66:1204-10.

25. Spagnolello O, Pinacchio C, Santinelli L, Vassalini P, Innocenti GP, De Girolamo G, Fabris S, Giovanetti M, Angeletti S, Russo A, Mastroianni CM, Ciccozzi M, Ceccarelli G, d'Ettorre G. Targeting microbiome: an alternative strategy for fighting SARS-CoV-2 infection. Chemotherapy. 2021. https://doi.org/10.1159/00051 5344 (Epub ahead of print).

26. Zuo T, Liu Q, Zhang F, Lui GC, Tso EY, Yeoh YK, Chen Z, Boon SS, Chan FK, Chan PK, Ng SC. Depicting SARS-CoV-2 faecal viral activity in association with gut microbiota composition in patients with COVID-19. Gut. 2021;70:276-84.

27. Welte T, Ambrose LJ, Sibbring GC, Sheikh S, Müllerová H, Sabir I. Current evidence for COVID-19 therapies: a systematic literature review. Eur Respir Rev. 2021;30: 200384.

28. Ko JJ, Wu C, Mehta N, Wald-Dickler N, Yang W, Qiao R. A comparison of methylprednisolone and dexamethasone in intensive care patients with COVID-19. J Intensive Care Med. 2021. https:// doi.org/10.1177/0885066621994057 (Epub ahead of print).

29. Tomazini BM, Maia IS, Cavalcanti AB, Berwanger O, Rosa RG, Veiga VC, Avezum A, Lopes RD, Bueno FR, Silva MVAO, Baldassare FP, Costa ELV, Moura RAB, Honorato MO, Costa AN, Damiani LP, Lisboa T, Kawano-Dourado L, Zampieri FG, Olivato GB, Righy C, Amendola CP, Roepke RML, Freitas DHM, Forte DN, Freitas FGR, Fernandes CCF, Melro LMG, Junior GFS, Morais DC, Zung S, Machado FR, Azevedo LCP, COALITION COVID-19 Brazil III Investigators. Effect of dexamethasone on days alive and ventilator-free in patients with moderate or severe acute respiratory distress syndrome and COVID-19: the CoDEX randomized clinical trial. JAMA. 2020;324:1307-16.

30. Garcia-Vidal C, Sanjuan G, Moreno-García E, Puerta-Alcalde P, Garcia-Pouton N, Chumbita M, Fernandez-Pittol M, Pitart C, Inciarte A, Bodro M, Morata L, Ambrosioni J, Grafia I, Meira F, Macaya I, Cardozo C, Casals C, Tellez A, Castro P, Marco F, García F, Mensa J, Martínez JA, Soriano A, COVID-19 
Researchers Group. Incidence of co-infections and superinfections in hospitalized patients with COVID-19: a retrospective cohort study. Clin Microbiol Infect. 2021;27:83-8.

31. Somers EC, Eschenauer GA, Troost JP, Golob JL, Gandhi TN, Wang L, Zhou N, Petty LA, Baang JH, Dillman NO, Frame D, Gregg KS, Kaul DR, Nagel J, Patel TS, Zhou S, Lauring AS, Hanauer DA, Martin E, Sharma P, Fung CM, Pogue JM. Tocilizumab for treatment of mechanically ventilated patients with COVID-19. Clin Infect Dis. 2020. https://doi.org/10.1093/cid/ ciaa954 (Epub ahead of print).
32. Russo A, Venditti M, Ceccarelli G, Mastroianni CM, d'Ettorre G. Procalcitonin in daily clinical practice: an evergreen tool also during a pandemic. Intern Emerg Med. 2021;16:541-3.

33. Russo A, Bellelli V, Ceccarelli G, Marincola Cattaneo F, Bianchi L, Pierro R, Russo R, Steffanina A, Pugliese F, Mastroianni CM, d'Ettorre G, Sabetta F. Comparison between hospitalized patients affected or not by COVID-19 (RESILIENCY study). Clin Infect Dis. 2020. https://doi.org/10.1093/cid/ciaa1745 (Epub ahead of print). 